\title{
PLUVIOMETRIA, BALANÇO HÍDRICO E PRODUTIVIDADE AGRÍCOLA PARA REGIÕES DA BAHIA E TOCANTINS
}

Iug Lopes ${ }^{1}$, Ailton Alves de Carvalho ${ }^{2}$, José Edson Florentino de Morais ${ }^{3}$, Douglas Alberto Silva ${ }^{4} \&$ Brauliro Gonçalves Leal $^{5}$

1 - Professor, Instituto Federal de Educação, Ciência e Tecnologia Baiano, Campus Bom Jesus da Lapa-BA, iug.lopes@ifbaiano.edu.br

2 - Doutorando, Universidade Federal Rural de Pernambuco, Departamento de Engenharia Agrícola, Recife-PE, ailtonalvesst@hotmail.com

3 - Doutorando, Universidade Federal Rural de Pernambuco, Departamento de Engenharia Agrícola, Recife-PE, joseedson50@hotmail.com

4 - Mestre, Universidade Federal Rural de Pernambuco, Departamento de Engenharia Agrícola, Recife-PE, douglasalbertosilva@hotmail.com

5 - Professor, Universidade Federal do Vale do São Francisco, Campus Juazeiro, Juazeiro-BA, brauliro.leal@univasf.edu.br

\section{Palavras-chave:}

Classificação pluviométrica

Técnica de Quantis

Yield Gap

Distribuição de Gumbel

\section{RESUMO}

Dentre as alterações que os índices pluviométricos proporcionam nas atividades, a mais vulnerável a essas mudanças é a agricultura. Dessa forma, o objetivo deste estudo foi verificar a qualidade dos dados pluviométricos anuais máximos no ajuste do modelo distribuição de Gumbel, realizar a estimativa de períodos de retorno para as precipitações máximas anuais, caracterizar os perfis da precipitação e, por meio da técnica de Quantis, demostrar a variabilidade temporal dos balanços hídricos climatológicos para microrregiões do estado da Bahia e de Tocantins, assim como verificar a dependência da produção agrícola com as precipitações. Com os dados de 1961 a 2016 para Barreiras e Taguatinga, foi possível construir o perfil histórico da precipitação, observando a ocorrência de eventos extremos máximos para a microrregião da Bahia e mínimos para a de Tocantins. A distribuição de probabilidade por Gumbel foi adequada na estimativa da intensidade de precipitação anual e suas frequências e permitiu observar que, a cada dois anos, pode ocorrer uma precipitação máxima anual igual ou superior a $1050 \mathrm{~mm}$ para Barreiras e 1400 mm para Taguatinga. O perfil histórico foi classificado através da técnica quantílica. Compararam-se os anos quantílicos com os dados de produção para as culturas de milho, soja e arroz. Houve uma tendência de aderência da precipitação anual máxima ao modelo probabilístico de Gumbel. Através do tempo de retorno foi possível observar que, a cada dois anos, existe a possibilidade de uma precipitação anual igual ou superior a 1050 e $1400 \mathrm{~mm}$ para Barreiras e Taguatinga, respectivamente. Ajustaram-se, por meio de modelo linear, as precipitações máximas anuais em relação ao aumento do período de retorno. É observada uma associação entre precipitação e produção agrícola, no período de análise para as localidades estudadas.

\section{Keywords:}

Precipitation classification Quantum technique

Yield Gap

Gumbel distribution
PLUVIOMETRY, WATER BALANCE AND AGRICULTURAL PRODUCTIVITY FOR BAHIA AND TOCANTINS REGIONS

\section{ABSTRACT}

Among the changes that pluviometric indices cause to activities, agriculture is the activity which is most vulnerable to these changes. Thus, the objective of this study was to verify the quality of the maximum annual rainfall data adjusting to the Gumbel distribution, to estimate the return periods for the annual maximum precipitation, to characterize the precipitation profiles and, through the Quantis technique, demonstrate the temporal variability of the climatological water balance for the micro-regions of the State of Bahia and Tocantins, as well as to verify the dependence of the agricultural production with the precipitations. Having data from 1961 to 2016 for Barreiras and Taguatinga it was possible to construct the historical precipitation profile while observing the occurrence of extreme maximum events for those micro-regions in Bahia and occurrence of minimum for those in Tocantins. Gumbel's probability distribution was adequate in the estimation of the annual precipitation intensity and its frequencies and allowed to observe that, every 2 years, a maximum annual precipitation equal to or greater than $1050 \mathrm{~mm}$ can occur for Barreiras and 1400 for Taguatinga. The historical profile was classified using the Quantum technique. He correlated the quantum years to the production data for cornbean, soybean and rice crops. There was a tendency of adherence of the maximum annual precipitation to Gumbel's probabilistic model. Through the time of return it was possible to observe that, every 2 years, there is the possibility of annual precipitation equal to or greater than 1050 and $1400 \mathrm{~mm}$ for Barreiras and Taguatinga, respectively. The maximum annual precipitations were adjusted by linear model in relation to the increase of the return period. An association between precipitation and agricultural production was observed during the period of analysis for the location in this study. 


\section{INTRODUÇÃO}

A análise do comportamento das chuvas tem grande importância devido à grande variabilidade espaço-temporal, não somente sob o enfoque climático e hídrico, mas também pelas consequências nas instâncias econômicas, sociais e culturais advindas (LUCENA et al., 2011).

Dentre as alterações que os índices pluviométricos proporcionam nas atividades, a mais vulnerável a essas mudanças é a agricultura (LOPES et al., 2016). De forma geral, as modificações no quantitativo interanual de precipitação podem afetar os sistemas agrícolas com sérias consequências na produção de alimentos em uma das maiores fronteiras agrícolas do país, Bahia e Tocantins (OLIVEIRA; BÜHLER, 2016).

Uma técnica de classificação pluviométrica é a de Quantis, que foi descrita por Xavier (2001) e tem sua fundamentação na distribuição da frequência acumulada. Para a aplicação dessa metodologia, que busca a aproximação da função densidade de probabilidade, quanto maior o número de observações melhor é a classificação.

A técnica quantílica possibilita delimitar valores entre faixas que classificam os diferenciados regimes pluviométricos: muito seco (MS), seco $(\mathrm{S})$, normal $(\mathrm{N})$, chuvoso (C) e muito chuvoso (MC). Santos e Aquino (2017) salientaram, no seu estudo, que a técnica é de aplicação simples, permitindo quantificar as ordens quantílicas, ou seja, suas disponibilidades hídricas anuais de uma dada região, utilizando-se apenas os dados pluviométricos.

Em balanços hídricos, principalmente de bacias hidrográficas, há uma grande incerteza que geralmente está associada à grandeza de um ou mais componentes. Por ilustração, têm-se o escoamento superficial e a evaporação, que são muitas vezes difíceis de medir/estimar de forma confiável e com alta precisão (TRASK et al., 2017).

Uma saída para balanços hídricos simplificados é o Balanço Hídrico Climatológico (BHC), que foi desenvolvido por Thornthwaite e Mather (1955). Esse balanço tem o objetivo de determinar o regime hídrico de determinada região local, sem que ocorra a utilização de medidas diretas das condições do solo.

Esse BHC tornou-se uma ferramenta de grande importância para avaliação e delimitação dos potenciais hídricos em nível regional, sendo aplicado na irrigação para verificação de excedentes ou escassez de água e na hidrologia para quantificação das disponibilidades hídricas, a exemplo de construção e manejo de reservatórios (AMORIM NETO, 1989).

A utilização dessa metodologia está sendo aprimorada e aplicada a novos cenários, a exemplo de Lopes et al. (2017a), que estudaram formas de planejamento hídrico através do BHC para se dimensionarem formas de manejos integrados dos recursos hídricos para anos com precipitação inferior, igual ou superior à normal anual.

A sazonalidade da relação entre o rendimento de grãos e o suprimento de água (água do solo disponível na semeadura, precipitação de semeadura e maturação e irrigação aplicada) já foi descrita pelo modelo Yield Gap. Assim, deve-se também realizar verificações em diversas culturas agrícolas e regiões (CASSMAN et al., 2015).

Dessa forma, o objetivo deste estudo foi verificar a qualidade dos dados pluviométricos anuais máximos no ajuste do modelo distribuição de Gumbel, realizar a estimativa de períodos de retorno para as precipitações máximas anuais, caracterizar os perfis da precipitação e, por meio da técnica de Quantis, demostrar a variabilidade temporal dos balanços hídricos climatológicos para microrregiões do estado da Bahia e de Tocantins, assim como verificar a dependência da produção agrícola com as precipitações das respectivas localidades.

\section{MATERIAL E MÉTODOS}

\section{ARÉA DE ESTUDO}

A região de estudo (Figura 1) compreende um polo agrícola com potencial e relevância no Brasil, representado pelo Extremo-Oeste da Bahia e pelo Oriental de Tocantins. Para a caracterização pluviométrica e do balanço hídrico, foram obtidos os dados de precipitação e temperatura através do site do Instituto Nacional de Meteorologia (www. 
inmet.gov.br/portal/).

Os dados foram de Estações Meteorológicas Convencionais, localizadas nos municípios de Barreiras (Código da Organização Mundial de Meteorologia: 83236; latitude: $12^{\circ} 9^{\prime} 0.0^{\prime \prime} \mathrm{S}$ e longitude: $45^{\circ} 0$ ' $36.0^{\prime \prime} \mathrm{W}$; altitude: $439,29 \mathrm{~m}$ ), inserido na microrregião de Barreiras e Taguatinga (Código da Organização Mundial de Meteorologia: 83235; latitude: $12^{\circ} 24^{\prime} 0.0^{\prime \prime} \mathrm{S}$ e longitude: $46^{\circ}$ 24 ' 36.0" W; altitude: $603,6 \mathrm{~m}$ ), inserido na microrregião de Dianópolis.

\section{OBTENÇÃODEDADOSECARACTERIZAÇÃO PLUVIOMÉTRICA}

Foram obtidos dados mensais de precipitação, convertidos, em seguida, em anuais para caracterização pluviométrica das regiões. $\mathrm{O}$ período utilizado compreendeu a série histórica de janeiro de 1961 a dezembro de 2016, total de 55 anos, atendendo, dessa forma, à recomendação da Organização Mundial de Meteorologia (OMM) de 30 anos ou mais de dados (WMO, 1989). Assim, admite-se a confiabilidade em conjunto de dados meteorológicos para caracterizar as regiões estudadas.

Após a caraterização pluviométrica dos dados anuais de precipitação máxima, realizou-se a aplicação do modelo de Gumbel (1958) e foi verificada a sua qualidade pelo teste de aderência de Kolmogorov-Smirnov (DAVID, 1958). Com as hipóteses "H0: a distribuição de Gumbel se ajusta aos dados" e "H1: a distribuição de Gumbel não se ajusta aos dados", obtiveram-se as devidas significâncias estatísticas através de planilhas e do software Sisvar 5.0 (FERREIRA, 2014).

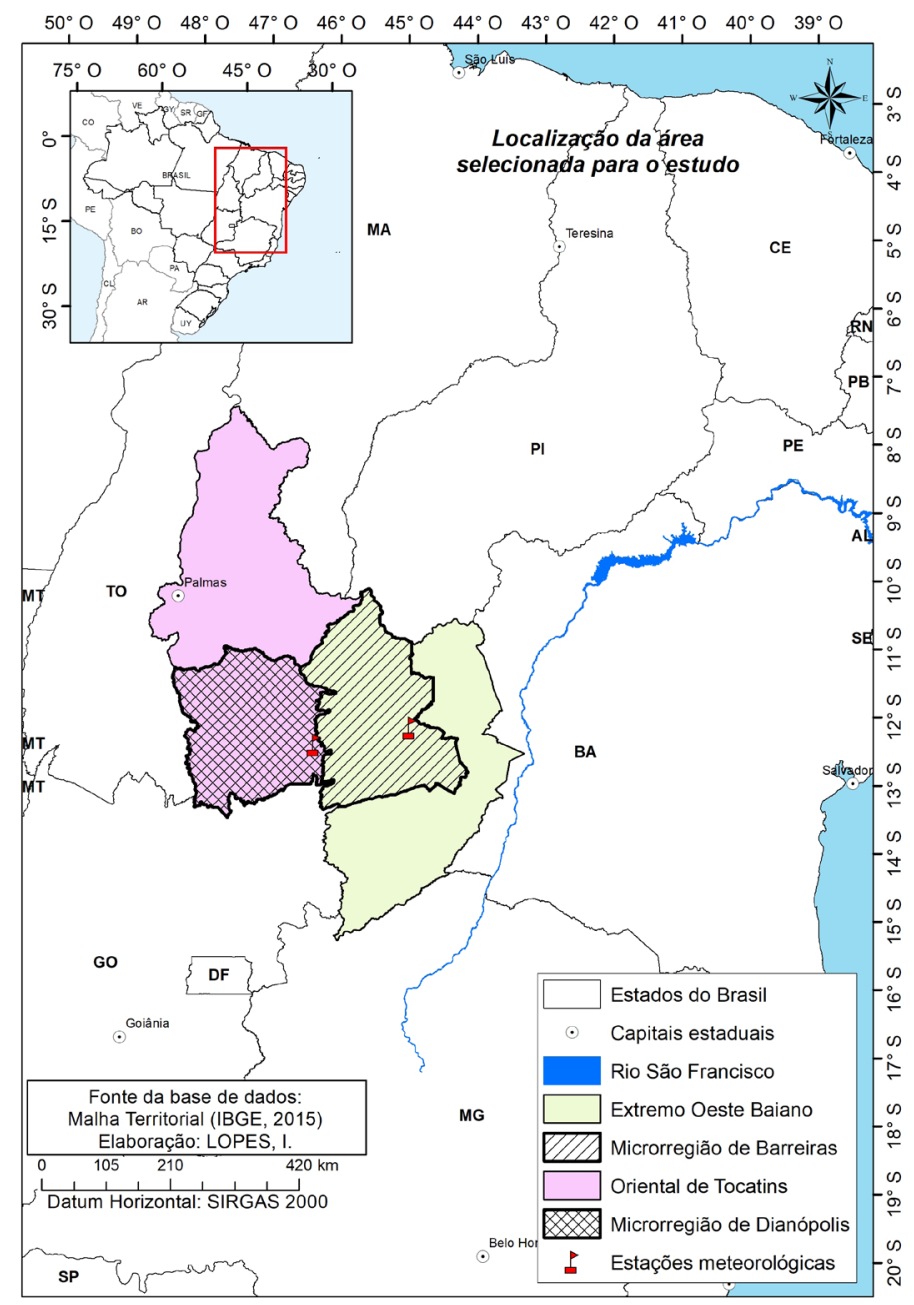

Fonte: Elaborada pelos autores.

Figura 1. Localização das microrregiões de estudo e suas respectivas estações meteorológicas do INMET 
$\mathrm{Na}$ continuidade da caracterização pluviométrica, após a verificação da aderência dos dados à distribuição de Gumbel, foi possível estimar períodos de retorno, para valores máximos dos acumulados anuais de precipitação em 2, 5, 10, 20, 30, 50, 100, 200 e 300 anos. Esses valores foram obtidos através da função de probabilidade acumulada anual da distribuição de Gumbel (Equação 1), permitindo assim a realização da função do tempo de retorno a partir da sua inversa (Equação 2).

$F y(y)=e^{-e^{-\left(\frac{-(y-\beta)}{\alpha}\right)}}$

$X(\log t)=\mu-\alpha^{*} \ln \left[-\ln \left(1-\frac{1}{\log t}\right)\right]$

em que,

$X(\log \mathrm{t})=$ precipitação máxima em função do tempo de retorno;

$\log \mathrm{t}=\log$ aritmo do tempo de retorno;

$\mu=$ parâmetro de forma; e

$\alpha=$ parâmetro de escala.

Para que as equações acima pudessem ser resolvidas, os parâmetros dessa distribuição de probabilidade foram estimados pelo Método dos Momentos conforme Equações 3 e 4 .

$\mu=x^{\prime}-0,451 * S$

$\alpha=\frac{1,2826}{s}$

em que,

$\mathrm{X}=$ média dos dados; e

$\mathrm{S}=$ desvio padrão.

Com a caracterização das precipitações máximas e tempo de recorrência, os dados foram ordenados anualmente para a aplicação da técnica de Quantis, que se baseia na frequência acumulada. Essa metodologia foi proposta por Xavier e Xavier (1987) e Xavier (2001), para avaliar a ocorrência dos anos secos e chuvosos sobre áreas extensas.

Nas regiões avaliadas neste estudo foram utilizados os Quantis para caracterização climática anual da precipitação, definidos nas categorias: muito seco (MS: $0-15 \%$ ), seco (S: $15-35 \%$ ), normal (N: $35-65 \%$ ), chuvoso (C: $65-85 \%$ ) e muito chuvoso (MC: $85-100 \%$ ). Os intervalos são estabelecidos para as classes extremas, sendo considerados como muito seco e muito chuvosos.

\section{CÁLCULO DO BALANÇO HÍDRICO}

Depois de classificados os anos nas classes pluviométricas, foram agrupados de acordo com o regime pluviométrico para determinação do balanço hídrico correspondente a cada classe. Essa metodologia de observação da disponibilidade de água nas respectivas classes pluviométricas foi primeiramente aplicada por Guimarães et al. (2017a), para estudar a região agrícola de Cruz das Almas-BA.

Para calcular o Balanço Hídrico Climatológico (BHC), utilizaram-se dados mensais de precipitação e de temperatura média condensada que foram adquiridos através do INMET. Assim, foram utilizados de forma direta no cálculo da metodologia proposta por Thornthwaite e Mather (1955). Seguindo para toda a série estudada, realizou-se o BHC para cada classe quantílica.

No software para o cálculo do balanço hídrico de Thornthwaite ${ }^{\circledR}$, a Evapotranspiração Potencial $(\mathrm{mm})$ é obtida pelas seguintes equações (Equações 5,6 e 7):

$\mathrm{ETP}=16,2^{*}\left[10^{*} \frac{\mathrm{T}}{\mathrm{I}}\right]^{\mathrm{a}} \quad\left(\mathrm{Se} 0 \leq \mathrm{T}<26,5^{\circ} \mathrm{C}\right)$

$\mathrm{a}=6,750 * 10-3 \mathrm{I}-7,711 * 10-5 \mathrm{I}+1,792 * 10-2 \mathrm{I}+0,492$

$\mathrm{ETP}=-415,85+32,24 * \mathrm{~T}-0,43^{*} \mathrm{~T}^{2} \quad\left(\mathrm{Se} \mathrm{T} \geq 26,5^{\circ} \mathrm{C}\right)$

sendo: o ETP correspondente à evapotranspiração potencial; Tà temperatura média do ar compensada $\left({ }^{\circ} \mathrm{C}\right)$; e I ao índice de calor (admensional). O valor do índice "a" é determinado em função do I, que 
foi obtido de acordo com as seguintes equações (Equações 8 e 9):

$\mathrm{I}=\sum \mathrm{i}_{\mathrm{n}}$

$\mathrm{i}=\left(\frac{\mathrm{Ta}}{5}\right)^{1,514}$

sendo: Ta correspondente à temperatura média do ar mês $\left({ }^{\circ} \mathrm{C}\right)$ e "i $\mathrm{i}_{\mathrm{n}}$ " ao índice térmico do mês " $n$ ".

O cálculo foi realizado com auxílio do software para o cálculo do balanço hídrico de Thornthwaite $^{\circledR}$. A Capacidade de Água Disponível (CAD) utilizada foi de $100 \mathrm{~mm}$.

\section{OBTENÇÃO DOS DADOS DE PRODUÇÃO AGRÍCOLA}

Com a obtenção da classificação dos anos em muito seco, seco, normal, chuvoso e muito chuvoso, com a técnica de Quantis, esses foram comparados com os dados de produtividade para as culturas de milho, soja e arroz. Sendo essas culturas as mais representativas para a região, em se tratando de área plantada e retorno econômico (SEPLAN, 2016; PAMPLONA et al., 2015).

A obtenção dos dados de produção agrícola para as microrregiões de Barreiras e Dianópolis foi através do Sistema Agropensa da Empresa Brasileira de Pesquisa Agropecuária (www. embrapa.br/agropensa), que apresenta produtos como o mapeamento e apoio à organização, integração e disseminação de base de dados e de informações agrícolas (EMBRAPA, 2013).

A variável referente à produtividade média observada para as microrregiões de Barreiras e Dianópolis que produzem, de forma representativa, milho, soja e arroz foi proveniente da razão entre a quantidade colhida (toneladas, t) e a área colhida (hectares, ha), portanto são expressas em $\mathrm{t} \mathrm{ha}^{-1}$. A análise da produtividade restringiu-se aos municípios que estão inseridos nas respectivas microrregiões.

Com precipitação correspondente à série histórica de 1961 a 2016 e os dados de produtividade das culturas de 1990 a 2015, foi possível comparar os dados de 1990 a 2015. Para verificação de influência de anos com chuvas abaixo do normal (muito secos e secos) e acima do normal (chuvosos e muito chuvosos), comparouse com a redução ou o aumento da produção, sendo que tenha ocorrido para as três culturas.

\section{RESULTADOS E DISCUSSÃO}

Com os dados de 1961 a 2016 para Barreiras e Taguatinga, foi possível construir o perfil histórico da precipitação e, assim, observa-se que, apesar de serem regiões próximas, possuem variações de precipitações distintas. Na estatística descritiva observada (Figura 2), constata-se que, na microrregião de Barreiras, ocorre uma menor precipitação média também nos seus valores de dispersão, quando comparada com a microrregião de Dianópolis.

Observou-se também a ocorrência de eventos extremos máximos de precipitação para a microrregião baiana e de mínimo para a de Tocantins. Esses eventos podem ser melhores observados na distribuição de Gumbel, assim como nos tempos de retorno para cada evento.

Para a caracterização da produção agrícola, pode-se observar o box-plot dos dados anuais para série histórica nas diferentes microrregiões entre os anos de 1990 e 2015 (Figura 3). A microrregião de Taguatinga apresentou uma maior produção para a cultura do milho, quando comparada com a de Barreiras.

$\mathrm{Na}$ Figura 4 podem ser percebidas as distribuições de frequência para a série das precipitações máximas anuais observadas e as estimadas pelo modelo de Gumbel, para a microrregião de Barreiras (Estação Meteorológica de Barreiras) e a de Dianópolis (Estação Meteorológica de Taguatinga). Para ambos os casos, observa-se boa aderência entre a distribuição observada e a teórica de Gumbel.

A distribuição de probabilidade por Gumbel foi adequada na estimativa da intensidade de precipitação anual e suas frequências, comprovada com a avaliação pelo teste de Kolmogorov- 


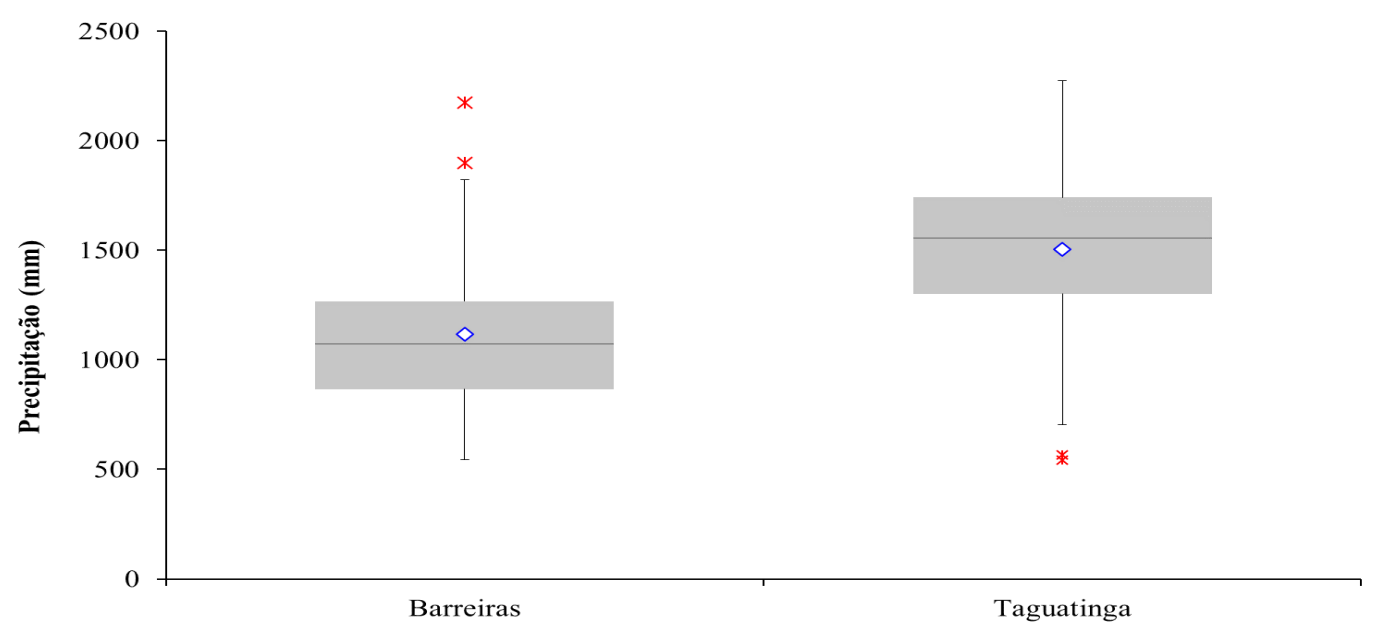

Legenda: $\diamond=$ média; $-=$ moda; $\square=25$ a $75 \%$ de probabilidade; $\perp=$ máximo e mínimo; $\mathrm{x}=1$ a $99 \%$ de probabilidade.

Figura 2. Box-plot dos dados de precipitação anual para série histórica nas estações localizada nas diferentes microrregiões entre os anos de 1961 e 2016

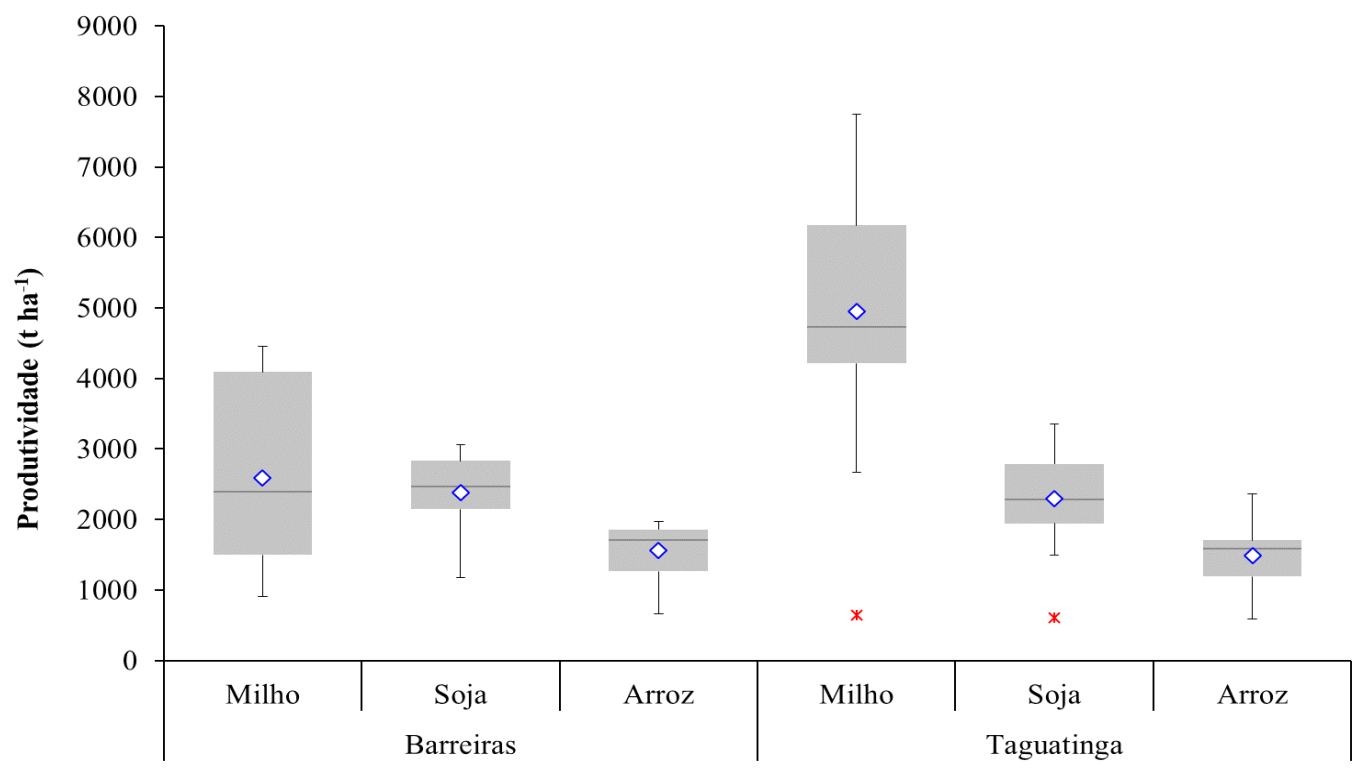

Legenda: $\diamond=$ média; - = moda; $\square=25$ a $75 \%$ de probabilidade,$\perp=$ máximo e mínimo; $\mathrm{x}=1$ a $99 \%$ de probabilidade.

Figura 3. Box-plot dos dados de produtividade anual para série histórica nas diferentes microrregiões entre os anos de 1990 e 2015

Smirnov ao nível de $1 \%$ de significância e com o uso de 47 dados para ambas as microrregiões.

Apesar das distribuições distintas em valores intermediários de precipitações para as microrregiões, ambas possuem valores de extremos semelhantes. Essa situação está diretamente relacionada com os outliers observados, sendo Barreiras para valores superiores e Taguatinga para valores inferiores da caracterização pluviométrica anual observado no box-plot.

Essa situação é semelhante a resultados 
- Barreiras $\square$ Taguatinga Gumbel

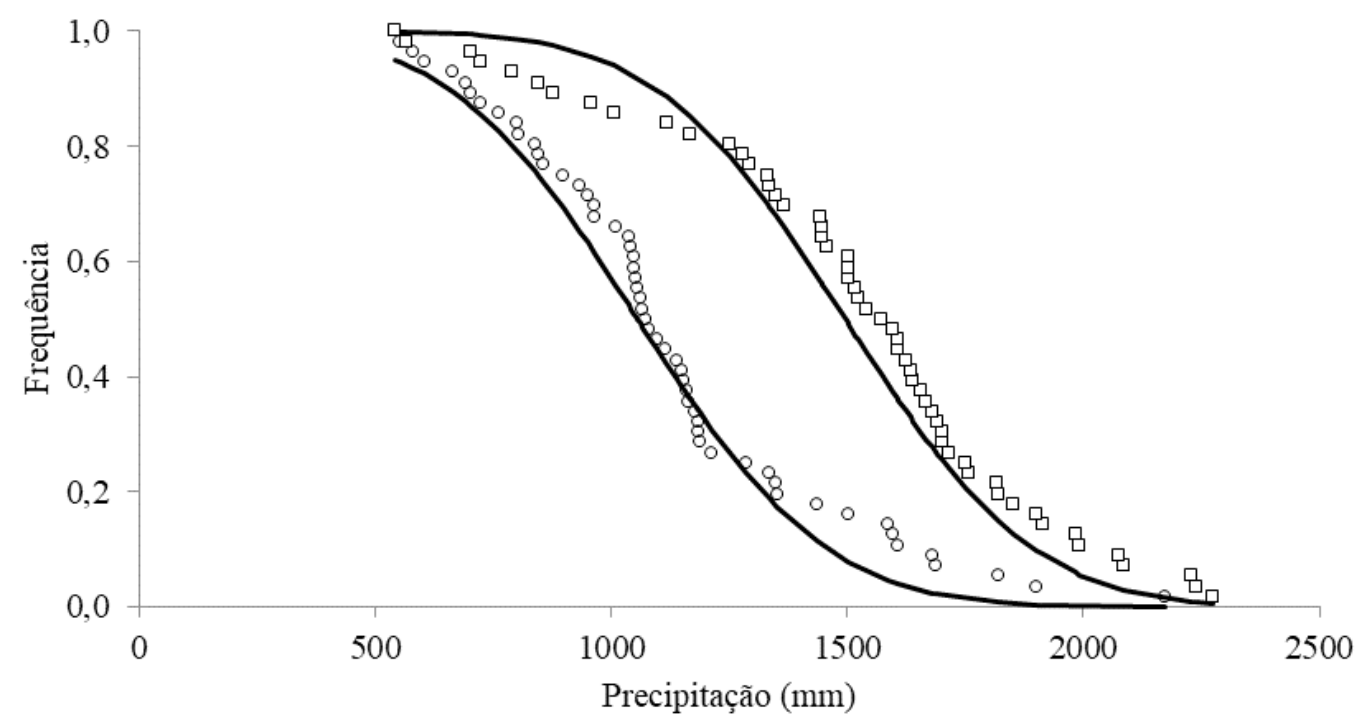

Figura 4. Distribuição de frequência das precipitações máximas anuais observadas e estimadas pelo modelo de Gumbel, para estações localizada nas diferentes microrregiões, entre os anos de 1961 e 2016.

obtidos por Monteiro et al. (2012), em que as distribuições pluviométricas anuais apresentaram curva suave e simétrica em relação ao pontos distribuídos, justificando assim a utilização de Gumbel de forma auxiliar à técnica de Quantis e comprovando a coerência do cálculo estatístico na obtenção dos valores normais e extremos.

Já na Figura 5, podem-se visualizar as estimativas das precipitações máximas anuais, em que são esperadas para diferentes períodos de retorno, em ano. O incremento das precipitações máximas anuais tende a se estabilizar com o aumento do período de retorno, ajustando-se, dessa forma, a um modelo logarítmico e assim linearizado, como observado.

Tem-se que, acada doisanos ( 0,3 correspondente a $\log \mathrm{t}$ ), pode ocorrer uma precipitação máxima anual igual ou superior a $1050 \mathrm{~mm}$ para Barreiras e $1400 \mathrm{~mm}$ para Taguatinga. Guimarães et al. (2017b) relataram que esse tempo de retorno para dois anos constitui-se um bom fator para o planejamento agrícola. Dessa forma, pode-se comparar com delineamento agroclimatológico, com culturas que se adequem ao regime pluviométrico caracterizado para as duas regiões estudadas.

Com a aplicação da metodologia quantílica de classificação pluviométrica anual, permite-se a visualização de pequena variabilidade das classes nas microrregiões estudadas. Esses resultados são de fundamental importância para planejamento de processos e etapas que necessitam de informações pluviométricas. Ao aplicar a técnica, pode-se constatar que a série histórica estudada possui uma precipitação anual média para os 55 anos de estudo (Q0,50).

A sua importância fica destacada com aplicação da metodologia pelo Centro Nacional de Monitoramento e Alertas de Desastres Naturais (CEMADEN, 2017), que a utilizou em várias regiões com a perspectiva de classificação pluviométrica para períodos de tempo distintos, uma vez que se destaca a variabilidade de precipitação e respectiva disponibilidade hídrica para todo o território nacional.

O estudo de precipitações em grandes volumes, em especial a distribuição de Gumbel, tem representado uma grande importância científica na análise de precipitações pluviais máximas e seus tempos de recorrência (BARRETO et al., 


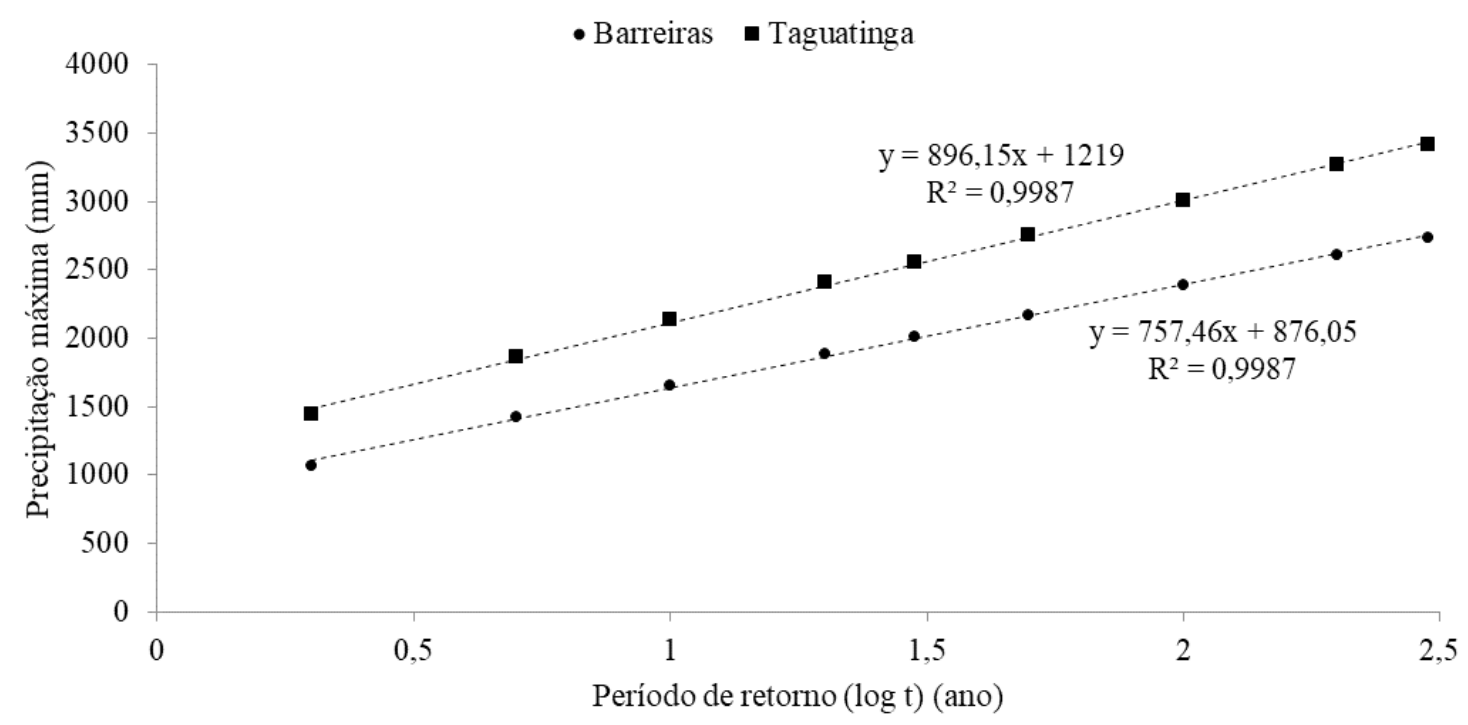

Figura 5. Precipitação máxima em função do período de retorno para estações localizadas nas diferentes mesorregiões entre os anos de 1961 e 2016

2015). Assim como alguns autores que avaliaram séries de dados de precipitação em diferentes localidades do Brasil (ALMEIDA et al., 2014; SANTOS et al., 2014), observou-se boa aderência entre a distribuição observada e a teórica obtida pelo modelo de Gumbel, para as microrregiões de Barreiras e de Dianópolis, já que há uma boa coerência entre os modelos com cada distribuição.

As duas microrregiões foram classificadas obtendo-se o perfil histórico da precipitação para o período de 1961 a 2016 (Figura 6A e 6B). Constatou-se muita variação no período, havendo anos seguidos com o mesmo regime de seco, normal, chuvoso e muito chuvoso para microrregião de Barreiras; já para a microrregião de Dianópolis ocorreram anos seguidos para todas as classificações quantílicas, porém não mais que dois anos para todas as classes da Dianópolis.

Com a obtenção mensal da precipitação e da temperatura, foi possível obter o BHC para a microrregião de Barreiras (Figura 7) e para a de Divinópolis (Figura 8). Observando o BHC do município de Barreiras para o período de 1961 a 2016, através dos valores dos déficits e dos excedentes, constata-se que o período chuvoso da microrregião tem seu início em outubro e perdura até o mês de março. Dessa forma, acumula $90 \%$ da sua precipitação anual total nos seis meses mencionados. Quando observado o período de menor disponibilidade de água no solo, tem-se que os meses de agosto e setembro são fortemente afetados por maior demanda evapotranspirativa da atmosfera.

Quando avaliados os meses em que ocorre a concentração pluviométrica para a microrregião de Barreiras, observa-se que acontece de outubro a março, sendo que, quando direcionada para a série histórica, há uma maior reposição no balanço hídrico de novembro a março.

Já quando observados para a microrregião de Divinópolis, os meses em que ocorre a concentração pluviométrica são de outubro a abril, e, quando observada a série histórica, há uma maior reposição no balanço hídrico de novembro a abril.

Com a construção dos BHC, podem-se observar diferentes padrões climáticos para as microrregiões de Barreiras e Divinópolis. As técnicas utilizadas neste estudo permitem observar que se tratam de áreas de transição na precipitação, com um menor valor para o Extremo-Oeste da Bahia, como observado no box-plot.

Além disso, o BHC permitiu verificar a estimativa anual para as diferentes classes 
PLUVIOMETRIA, BALANÇO HÍDRICO E PRODUTIVIDADE AGRÍCOLA PARA REGIÕES DA BAHIA E TOCANTINS

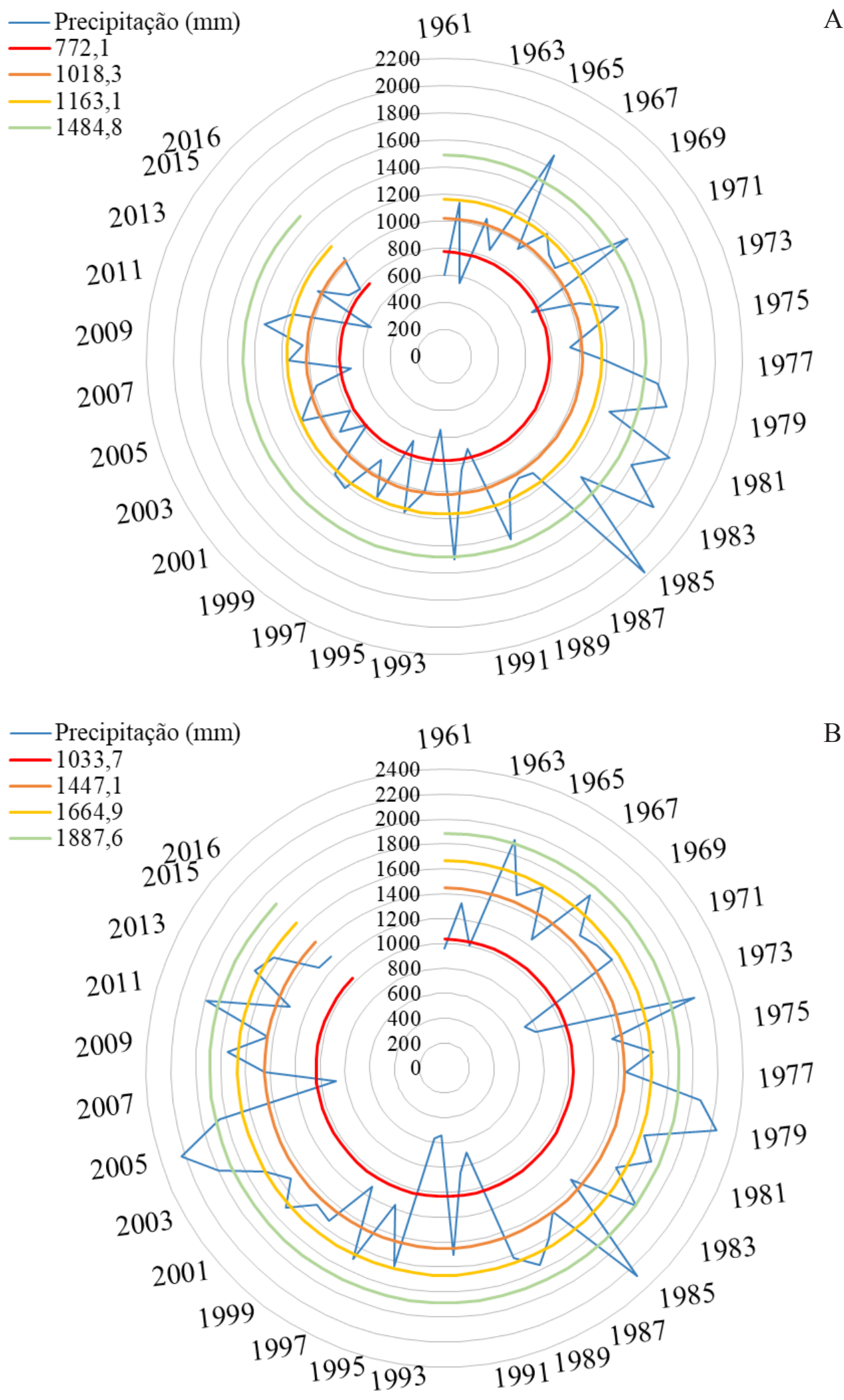

Figura 6. Classificação do perfil histórico da precipitação anual $(\mathrm{mm})$ de acordo com a classificação da técnica de Quantis - as linhas correspondem à divisão entre muito seco e seco (1033,7 mm); seco e normal (1447,0 mm); normal e chuvoso (1664,8 mm); chuvoso e muito chuvoso (1887,6 $\mathrm{mm})$ para as microrregiões de (A) Barreiras e de (B) Taguatinga no período de 1961 a 2016 


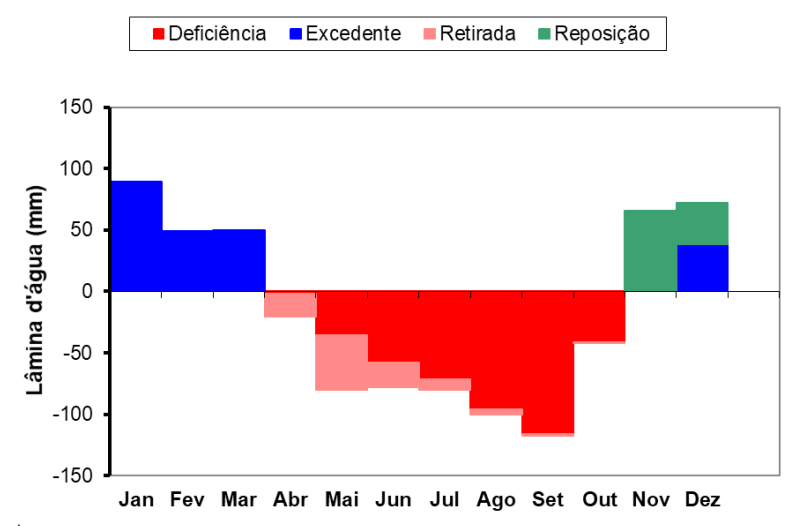

A

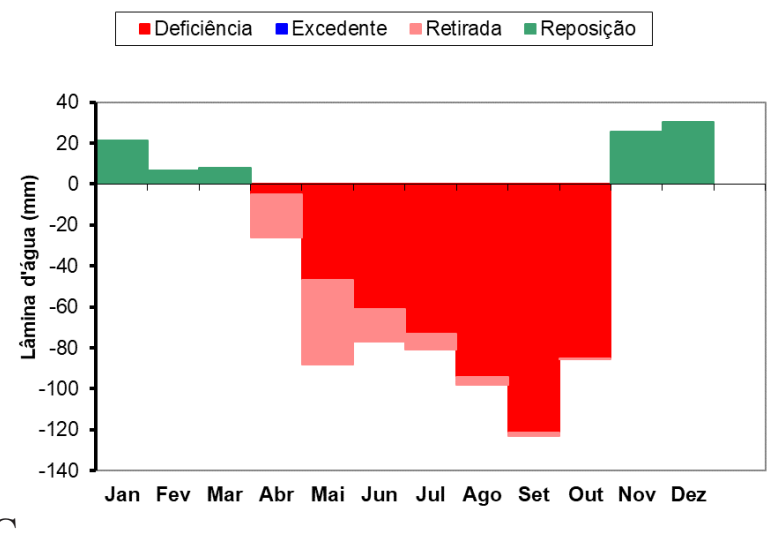

Deficiência - Excedente "Retirada =Reposição

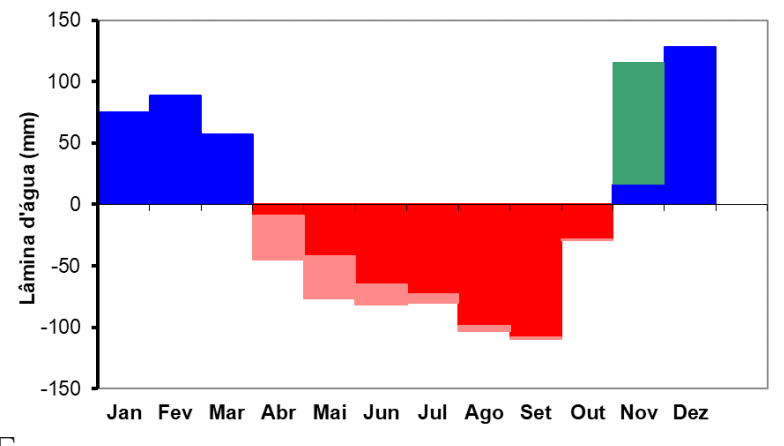

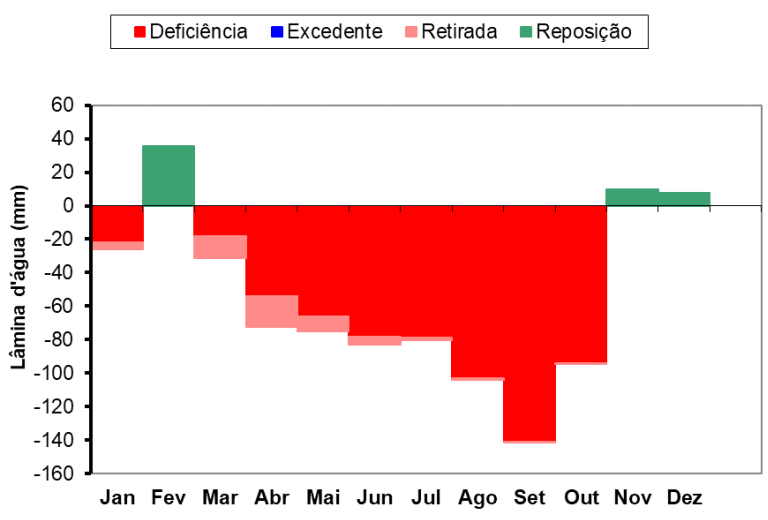

$\mathrm{B}$
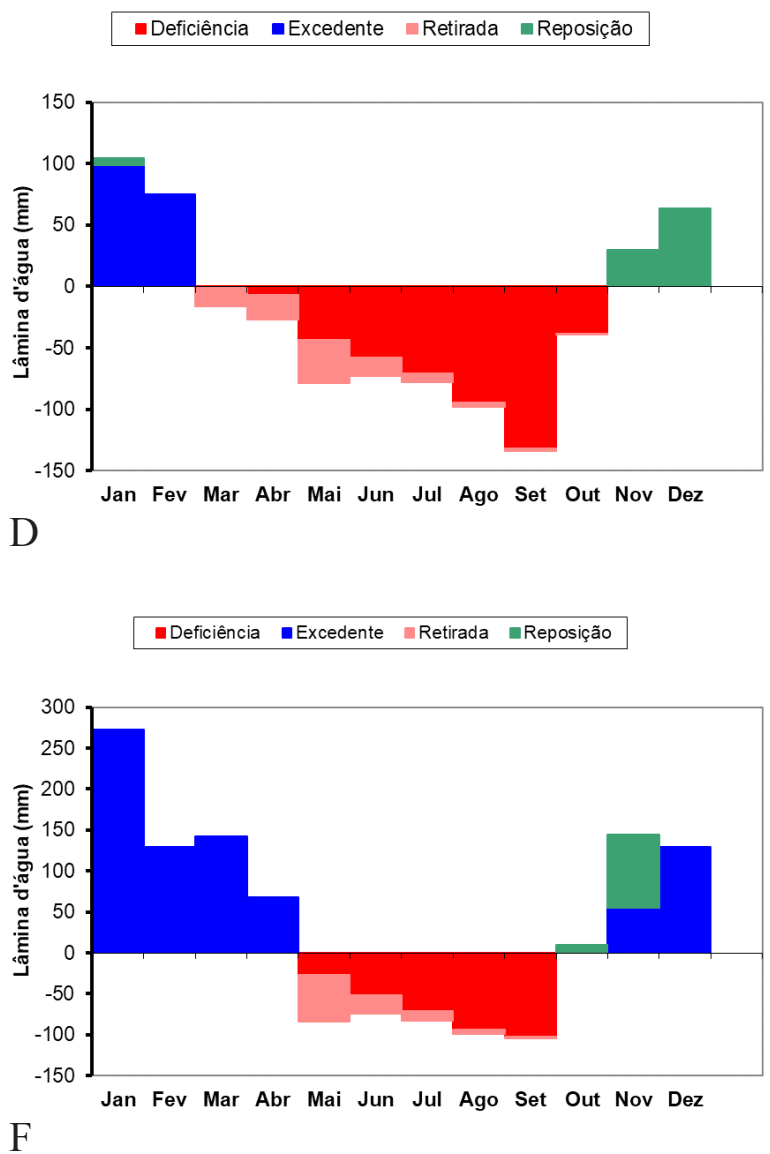

Figura 7. Balanço hídrico para a série histórica (A) e os regimes pluviométricos muito seco (B), seco (C), normal (D), chuvoso (E) e muito chuvoso (F) para a mesorregião do Sertão de Pernambuco - os eixos verticais não se encontram na mesma magnitude

quantílicas, que ocorrem de forma bastante variável, e com pequena quantidade de dados de elementos meteorológicos para entrada. De forma similar, foi observado por Lopes et al. (2017b), para diferentes mesorregiões do estado de Pernambuco, em que, com informações dos balanços hídrico climatológico de forma distribuída ao longo do estado, foi possível adotar medidas preventivas de manutenção de umidade do solo e de manejo de água excedente no solo.

Com os resultados obtidos do BHC de Thornthwaite e Mather (1955), observou-se 


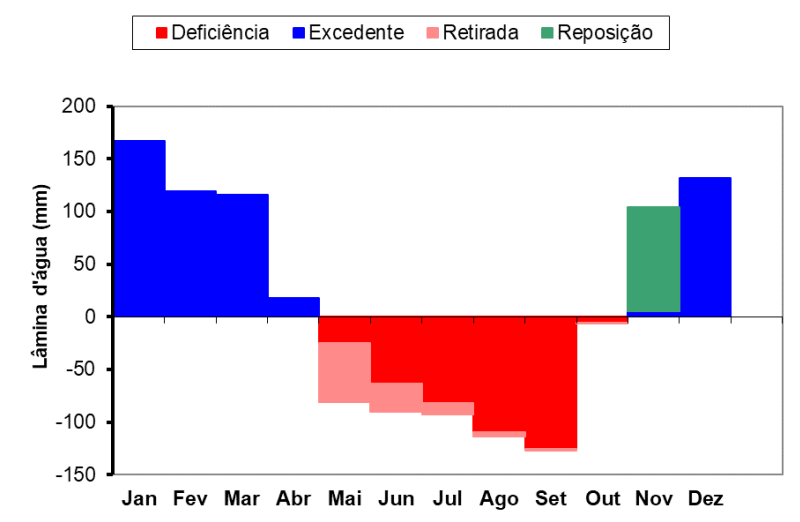

A

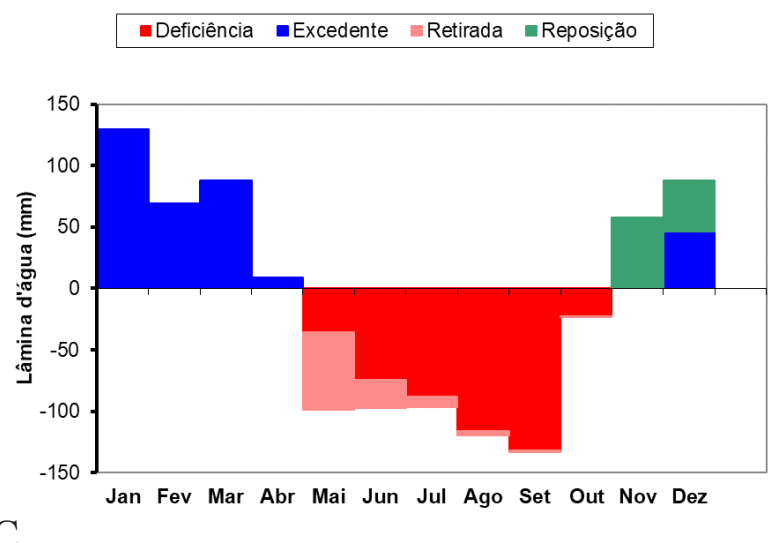

C

Deficiência Excedente $\quad$ Retirada $\quad$ Reposição

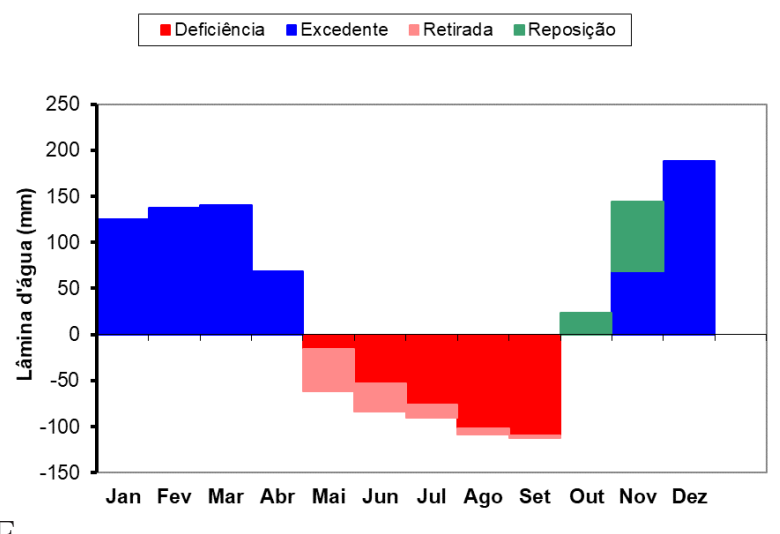

$\mathrm{E}$

Figura 8. Balanço hídrico para a série histórica (A) e os regimes pluviométricos muito seco (B), seco (C), normal (D), chuvoso (E) e muito chuvoso (F) para a mesorregião do Sertão de Pernambuco - os eixos verticais não se encontram na mesma magnitude

que tem apresentado qualidade que possibilita a caracterização em zoneamentos agroclimáticos, além de características como necessidade de água para irrigação e classificação climática. Tal magnitude aumenta a aplicabilidade dos resultados obtidos e pode, assim, ser usado para comparação
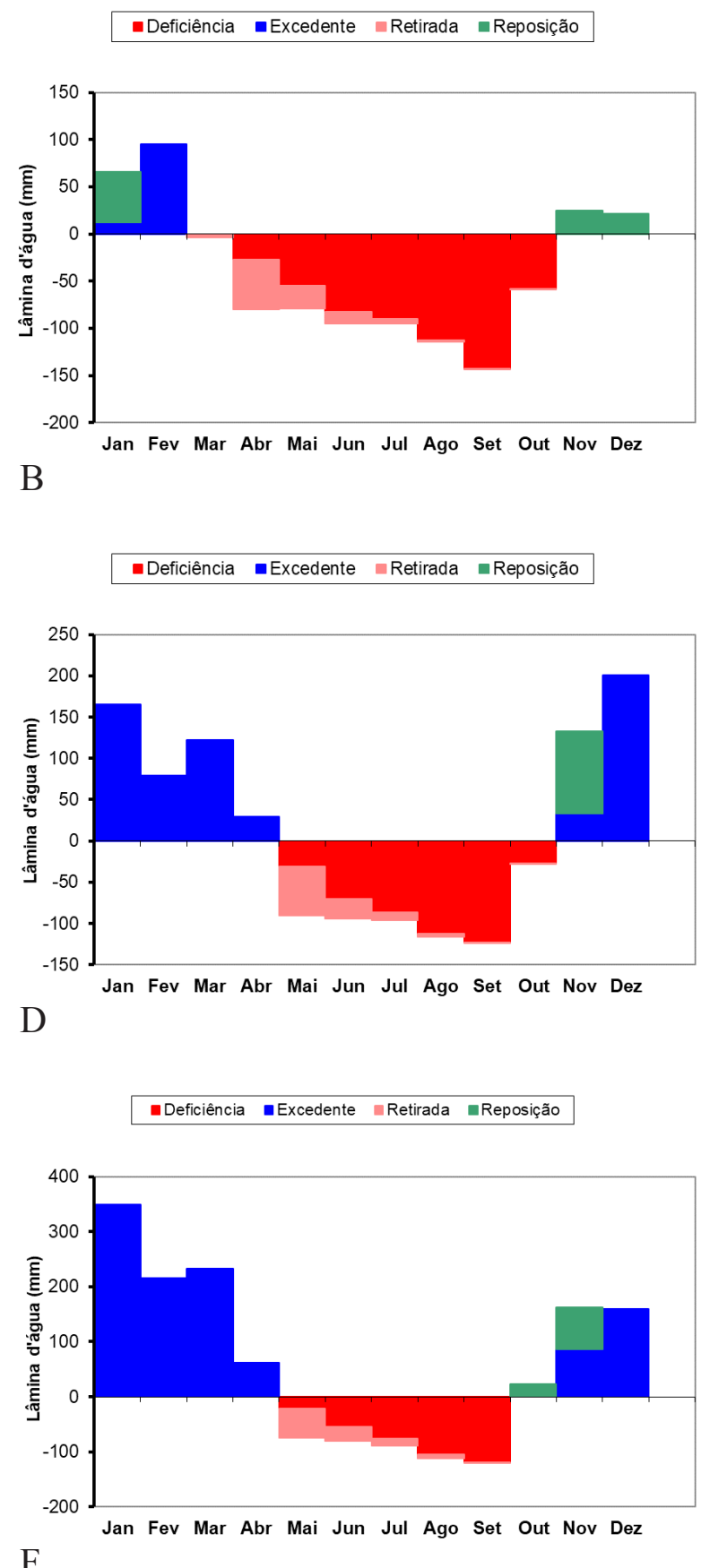
de produção para as culturas de milho, soja e arroz, mais representativas da região (Figura 9). A associação entre dados climáticas (classes quantílicas) e dados de precipitação foram boas, e, de forma quantitativa, pode-se observar 8 para a microrregião de Barreiras e 9 para de Taguatinga.

As correlações existentes entre regimes pluviométricos e as produtividades agrícolas é subtendida. Porém, Silva et al. (2008) relataram que alguns fatores que estão próximo ao nível da precipitação é o desenvolvimento da cultura adotada para determinada região e do grau de tecnologia empregada. Relataram ainda que quanto menor for a utilização de tecnologias, assim como o planejamento agrícola, maiores serão as influências no âmbito negativo das variações dos regimes pluviométricos.

Com as observações de redução ou aumento de produtividade através da chuva, de 8 para a microrregião de Barreiras e 9 para a de Taguatinga, tem-se que o real efeito da precipitação na produção agrícola está abundantemente interligado com a época em que ocorrem os eventos, ou seja, sua distribuição durante o ciclo da cultura.
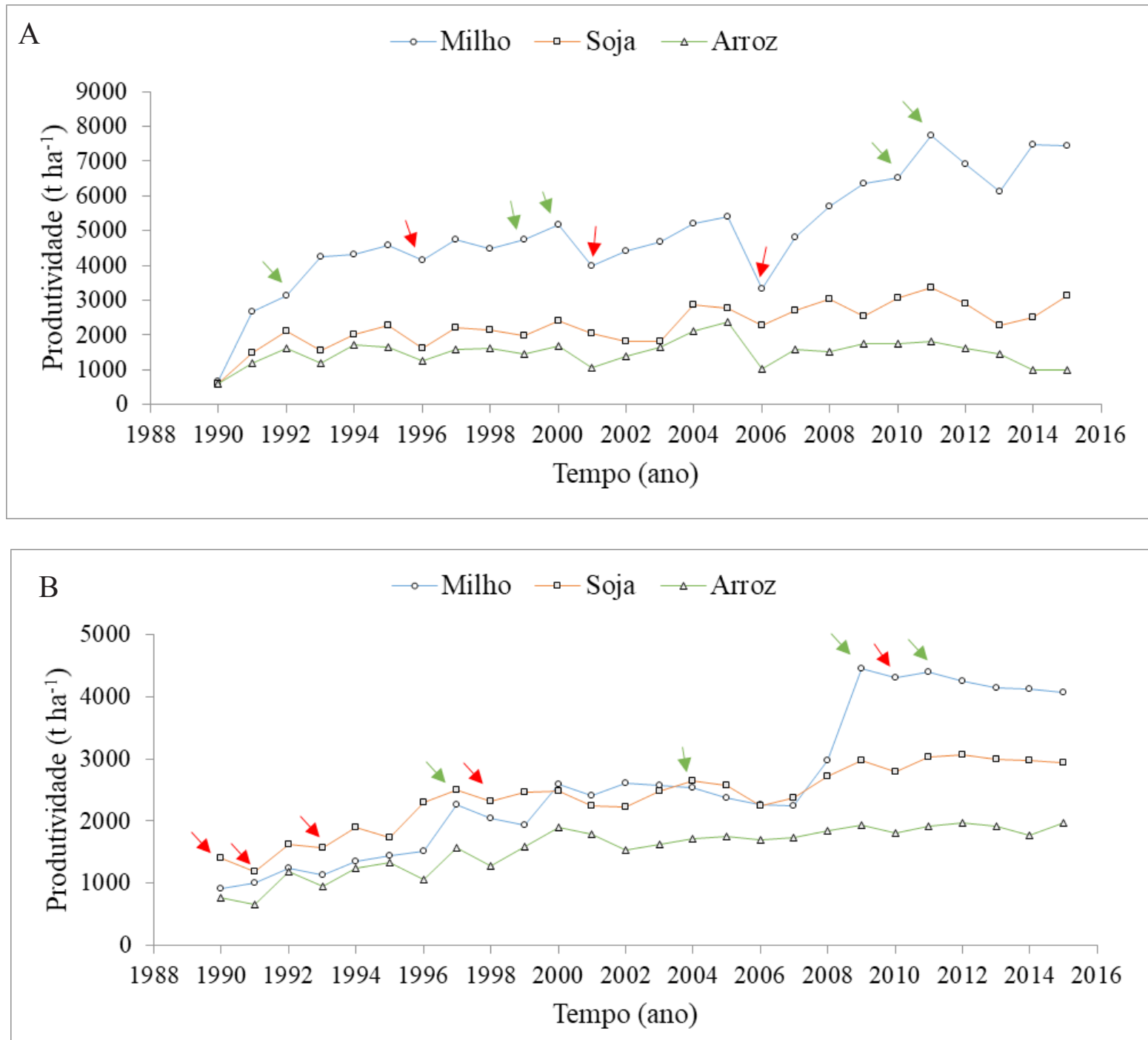

Figura 9. Produtividade anual das culturas de milho, soja e arroz para as microrregiões de (A) Barreiras e de (B) Dianópolis e comparadas com a classificação quantílica, sendo que a seta verde indica os anos em que ocorreram elevação da produtividade e precipitações acima do normal, e a seta vermelha a redução da produtividade e chuva abaixo do normal 
Apesar das perspectivas de correlação da produção com a precipitação, observou-se, para as duas microrregiões, uma tendência significativa de aumento da produtividade média das culturas de milho, soja e arroz ao longo do período analisado, e que estão diretamente relacionadas à melhoria das cultivares, tratos culturais e tecnologia aplicada.

\section{CONCLUSÕES}

- O modelo probabilístico de Gumbel tem boa aderência aos dados de precipitação anual máxima para as duas localidades. Observa-se que, a cada dois anos, existe a possibilidade de uma precipitação pluvial anual igual ou superior a $1050 \mathrm{~mm}$ para Barreiras e 1400 para Taguatinga.

- As precipitações máximas anuais das localidades estudadas se ajustaram por meio de um modelo linear em relação ao aumento do período de retorno. $\mathrm{Na}$ associação quantitativa entre precipitação e produção agrícola, de 1990 a 2015, observa-se 8 para a microrregião de Barreiras e de 9 para a de Taguatinga.

- O entendimento da precipitação diante da produtividade pode, também, ser realizado de forma sub-anual, sendo explicado pelo fato das diversas culturas agrícolas possuírem demandas hídricas e diferentes sensibilidades ao estresse hídrico, em cada etapa fenológica.

\section{REFERÊNCIAS BIBLIOGRÁFICAS}

AMORIM NETO, M.S. Balanço hídrico segundo Thornthwaite \& Mather (1955). EMBRAPACPATSA, 1989. 18p.

ALMEIDA, B.M.; JOSÉ, J.V.; DUARTE, S.N.; FRIZZONE, J.A.; ARRAES, F.D.; PERBONE, A. Análise de tendência temporal da precipitação diária máxima no Estado de São Paulo. Water Resources and Irrigation Management, Cruz das Almas, v.3, p.1-12, 2014.

AMORIM NETO, M.S. Balanço hídrico segundo Thornthwaite \& Mather (1955). (EMBRAPA CPTSA, Petrolina). n.34, p.1-18, 1989.

BARRETO, H.B.F.; PEREIRA, G.M.; BARRETO, F.P.; FREIRE, F.G.et alC.; MAIA, P.M.E. Relação intensidade-duração-frequência para precipitação extrema em Mossoró - RN. Global Science and Technology, Goiás, v.7, n.2, 2015.

CASSMAN, K.G.G.; GRASSINI, P.; HALL, A.J.; BASTIAANSSEN, W.G.M.; LABORTE, A.G.; MILNE, A.E.; SILESHI, G.W.; STEDUTO, P. Yield gap analysis of field crops: methods and case studies. FAO Water Reports, n.41, Rome, Italy, 2015.

DAVID, H.T.A Three-Sample KolmogorovSmirnov Test. The Annals of Mathematical Statistics, v.29, n.3, p.842-851, 1958

EMBRAPA - SISTEMA AGROPENSA DA EMPRESA BRASILEIRA DE PESQUISA AGROPECUÁRIA.. Produção Agrícola municipal. Disponível em: <https://www. embrapa.br/agropensa/producao-agricolamunicipal>. Acesso em: 20/12/2013.

FERREIRA, D.F. Sisvar: a Guide for its Bootstrap procedures in multiple comparisons. Ciência $\mathbf{e}$ Agrotecnologia, Lavras, v.38, p.109-112, 2014

GUIMARÃES, M.J.M.; LOPES, I.; OLDONI, H.; FILHO, M.A.C. Balanço hídrico para diferentes regimes pluviométricos na região de Cruz das Almas-BA. Revista de Ciências Agrárias/Amazonian Journal of Agricultural and Environmental Science, Amazônia v.59, p. 252-258, 2017a.

GUIMARÃES, M.J.M.; LOPES, I.; SILVA, W.P. da; SILVA, A.S. da. Estudo das precipitações máximas para o município de Cruz das Almas, 
BA. MAGISTRA, Cruz das Almas, v.27, p.316322, $2017 b$.

GUMBEL, E.J. Statistics of Extremes (New York: Columbia University Press). 1958. 338p.

LOPES, I.; GUIMARÃES, M.J.M.; MELO, J.M.M. de; RAMOS, C.M.C. Balanço hídrico em função de regimes pluviométricos na região de Petrolina-PE. IRRIGA, Botucatu, v.22, p.443457, $2017 \mathrm{a}$.

LOPES, I.; LEAL, B.G.; RAMOS, C.M.C.; MELO, J.M.M. DE Espacialização da precipitação para a região do Submédio São Francisco. Revista Brasileira de Agricultura Irrigada, v.10, 893903, 2016.

LOPES, I.; LEAL, B.G.L. Software para o cálculo do balanço hídrico de Thornthwaite Patente BR 512016 001060-6. 2016.

LOPES, I.; MELO, J.M.M.; MONTENEGRO, A. A.A.; GUIMARAES, M.J.M.; LOPES, B.; LEAL, B.G. Caracterização pluviométrica, precipitações máximas e balanço hídrico para diferentes regimes pluviométricos em mesoregiões de Pernambuco. REVISTA BRASILEIRA DE AGROTECNOLOGIA, v.7, p.20-33, 2017b.

LUCENA, D.B.; FILHO, G.F.M.; SERVAIN, J. Impact evaluation of the extreme climatic events in the pacific and atlantic oceans on the northeast Brazil rainy season. Revista Brasileira de Meteorologia, São José dos Campos, v.26, 297312, 2011.

MONTEIRO, J.B.; ROCHA, A.B.; ZANELLA, M.E. Técnica dos quantis para caracterização de anos secos e chuvosos (1980-2009): baixo curso do Apodi-Mossoró/RN. Revista Geográfica Acadêmica, Roraima, v.23, p.232-249, 2012.

OLIVEIRA, V.L. de; BÜHLER, È.A. Técnica e natureza no desenvolvimento do "Agronegócio."
Caderno CRH, Salvador, v.29, n.77, p.261-280, 2016.

PAMPLONA, C.; STAHLKE, L.; FÉLIX, C. Cerrado da Bahia: 40 anos. AIBA Rural, 2015. $36 \mathrm{p}$.

SANTOS, F. A. dos; AQUINO, C.M.S. de. Análise da precipitação pluviométrica no município de Castelo do Piauí, Nordeste do Brasil. GEOUSP Espaço e Tempo, São Paulo, v.21, n.2, p.619633, 2017.

SANTOS, W.O.; MESQUITA, F.O.; BATISTA, B.D.O.,;BATISTA, R.O.; ALVES, A.S. PRECIPITAÇÕES MÁXIMAS PARA O MUNICÍPIO DE MOSSORÓ DE 1964 A 2011 PELA DISTRIBUIÇÃO DE GUMBEL. IRRIGA, Botucatu, v.19, n.2, p.207-213, 2014.

SEPLAN. Base de dados Geográficos: atualização 2015. 2016.

SILVA, D.F.; PRELA-PANTANO, A.; NETO, J.L.S.A. Variabilidade da precipitação e produtividade agrícola na região do Médio Paranapanema, SP. Revista Brasileira Climatologia, Curitiba, v.3, p.101-116, 2008.

THORNTHWAITE, C.W.; MATHER, J.R. The water balance (Centerton, New Jersey: Drexel Institute of Technology, Laboratory of Climatology), v.8, n.1, 1955, 104p.

TRASK, J.C.; FOGG, G.E.; PUENTE, C.E. Resolving hydrologic water balances through a novel error analysis approach, with application to the Tahoe basin. Journal of Hydrology, Estados Unidos, v.546, 326-340, 2017.

WMO - World Meteorological Organization. Calculation of monthly and annual 30-year standard normals: prepared by a Meeting of Experts, Washington, D.C., USA, March 1989. (Geneva: WMO). 1989. 
PLUVIOMETRIA, BALANÇO HÍDRICO E PRODUTIVIDADE AGRÍCOLA PARA REGIÕES DA BAHIA E TOCANTINS

XAVIER, T.M.B.S. Tempo de Chuva - Estudos Climáticos e de Previsão para o Ceará e Nordeste Setentrional, ABC Editora, FortalezaCeará. 2001, 478p.
XAVIER, T.M.B.S.; XAVIER, A.F.S. Classificação e monitoramento de períodos secos ou chuvosos e cálculo de índices pluviométricos para a região nordeste do Brasil. Revista Brasileira de Engenharia, v.5, n.2, 1987. 